\title{
CENTRAL ROTATIONS OF MILKY WAY GLOBULAR CLUSTERS*
}

\author{
Maximilian H. Fabricius ${ }^{1,2}$, Eva Noyola ${ }^{3}$, Surangkhana Rukdee ${ }^{1}$, Roberto P. Saglia ${ }^{1}$, Ralf Bender ${ }^{1,2}$, \\ Ulrich Hopp $^{1,2}$, Jens Thomas ${ }^{1}$, Michael Opitsch ${ }^{2,4}$, and Michael J. Williams ${ }^{1,5}$ \\ ${ }^{1}$ Max Planck Institute for Extraterrestrial Physics, Giessenbachstrasse, D-85748 Garching, Germany; mxhf@mpe.mpg.de \\ ${ }^{2}$ University Observatory Munich, Scheinerstraße 1, D-81679 Munich, Germany \\ ${ }^{3}$ McDonald Observatory, The University of Texas at Austin, 2515 Speedway, Stop C1402, Austin, TX 78712-1206, USA \\ ${ }^{4}$ Excellence Cluster Universe, Boltzmannstr. 2, D-85748 Garching, Germany \\ ${ }^{5}$ Department of Astronomy, Columbia University, NY 10027, USA \\ Received 2014 March 31; accepted 2014 May 2; published 2014 May 14
}

\begin{abstract}
Most Milky Way globular clusters (GCs) exhibit measurable flattening, even if on a very low level. Both cluster rotation and tidal fields are thought to cause this flattening. Nevertheless, rotation has only been confirmed in a handful of GCs, based mostly on individual radial velocities at large radii. We are conducting a survey of the central kinematics of Galactic GCs using the new Integral Field Unit instrument VIRUS-W. We detect rotation in all 11 GCs that we have observed so far, rendering it likely that a large majority of the Milky Way GCs rotate. We use published catalogs of GCs to derive central ellipticities and position angles. We show that in all cases where the central ellipticity permits an accurate measurement of the position angle, those angles are in excellent agreement with the kinematic position angles that we derive from the VIRUS-W velocity fields. We find an unexpected tight correlation between central rotation and outer ellipticity, indicating that rotation drives flattening for the objects in our sample. We also find a tight correlation between central rotation and published values for the central velocity dispersion, most likely due to rotation impacting the old dispersion measurements.
\end{abstract}

Key words: globular clusters: general - stars: kinematics and dynamics - techniques: imaging spectroscopy

\section{INTRODUCTION}

Globular clusters (GCs) have historically been viewed as simple systems whose evolutionary history is well understood, but new observations keep revealing surprising results. GCs have short central relaxation times compared to their ages. Anisotropies and rotation are therefore likely to be very small in the central regions, while a moderate amount is expected in the outskirts (Meylan \& Heggie 1997).

Flattening (with a median axial ratio $\approx 0.9$ ) is found in the outer regions of Galactic GCs (White \& Shawl 1987; Chen \& Chen 2010). Clusters closer to the bulge tend to be more flattened than those in the halo. Given this observed flattening, rotation is expected in the outer parts of GCs, and indeed it has been measured for a number of them. Table 7.2 in Meylan \& Heggie (1997) includes all the relevant references before 1997. More recent work by Lane et al. (2011) and Bellazzini et al. (2012) and references in Tables 1 and 2 of Zocchi et al. (2012) use individual radial velocity measurements to show that anisotropy increases toward the outskirts of clusters. It is still under debate how much of the observed flattening seen for the outer parts of GCs is due to galactic tidal effects, disk cross shocking or to rotation.

On the theory side, analytical (Longaretti \& Lagoute 1997), Fokker-Planck (Einsel \& Spurzem 1999), and $N$-body models (Ernst et al. 2007) indicate that the presence of rotation affects the dynamical evolution of single mass star clusters, by accelerating core-collapse timescales. The caveat is that the effect seems to vanish for isolated two-mass $N$-body models (Ernst et al. 2007). Recent models indicate that rotation could be a key ingredient in the formation of multiple generations of stars in GCs (Bekki 2010; Mastrobuono-Battisti \& Perets 2013). The time evolution of $N$-body models seems to indicate that the rotation signature for the central region gets erased after a few

\footnotetext{
* This Letter includes data taken at The McDonald Observatory of The University of Texas at Austin.
}

relaxation times (Figure 14 of Ernst et al. 2007), which implies that rotation is not expected around the core radius of relaxed GCs.

Detailed modeling including central kinematics has been performed for a few clusters. In particular, $\omega$ Cen (van Leeuwen \& Le Poole 2002), 47 Tuc (Anderson \& King 2003), and M15 (van den Bosch et al. 2006) have been observed to rotate using various data sets and analysis techniques. Bianchini et al. (2013) undertakes the most thorough dynamical modeling for these three clusters to date. They find that for all three clusters, which have very different dynamical states, the ellipticity and anisotropy decrease toward the center, to the point of being practically isotropic inside the core radius. Besides these three cases, kinematic observations covering the regions around one or two core radii are scarce.

We are collecting optical, high resolution Integral Field Unit (IFU) spectroscopy of Milky Way GCs in an ongoing survey. In this Letter we present data for the first 11 clusters that we have observed.

\section{SAMPLE AND OBSERVATIONS}

Our sample was constructed based only on feasibility: we first selected all objects that are observable from the McDonald Observatory (at airmass <2) from the catalog of Milky Way GCs by Harris (1996, hereafter H96) and for which surface brightness estimates (Trager et al. 1995; Noyola \& Gebhardt $2006)$ led to reasonable exposure times $(<6 \mathrm{hr})$. This gave a sample of 27 clusters, 11 of which are presented here.

The observations were carried out during 2012 August, and 2013 April and August using the fiber-based IFU Spectrograph VIRUS-W (Fabricius et al. 2012) at the $2.7 \mathrm{~m}$ Harlan J. Smith Telescope of the McDonald Observatory in Texas. We used the higher resolution mode of the instrument with $R \sim 9000$ and a wavelength coverage of $4855-5475 \AA$. The IFU has a field of view of $105^{\prime \prime} \times 55^{\prime \prime}$ with the long edge always aligned with the 
Table 1

Central Rotation Gradients, Kinematic and Photometric Position Angles, and Ellipticities

\begin{tabular}{|c|c|c|c|c|c|c|c|}
\hline Identifier & $\begin{array}{c}t_{\text {obs, total }} \\
\text { (hr) } \\
\text { (2) }\end{array}$ & $\begin{array}{c}\text { R.A. } \\
\text { (J2000) } \\
(3)\end{array}$ & $\begin{array}{c}\text { Decl. } \\
(\mathrm{J} 2000) \\
(4)\end{array}$ & $\begin{array}{c}\|\nabla v\| \\
\left(\mathrm{km} \mathrm{s}^{-1} \operatorname{arcmin}^{-1}\right) \\
(5)\end{array}$ & $\begin{array}{l}\text { P.A.kin } \\
\text { (deg.) } \\
(6)\end{array}$ & $\begin{array}{l}\text { P.A.phot } \\
\text { (deg.) } \\
\text { (7) }\end{array}$ & $\begin{array}{c}100 \times \epsilon_{\mathrm{phot}}^{r<100^{\prime \prime}} \\
(8)\end{array}$ \\
\hline M3 & 1.7 & $13: 42: 11.598$ & $+28: 22: 37.94$ & $1.5 \pm 0.1 \pm 0.1$ & $102.2 \pm 4.5 \pm 11.8$ & $79.8 \pm 2.8 \pm 18.0$ & $2.2 \pm 0.2 \pm 1.1$ \\
\hline M5 & 3.3 & $15: 18: 33.143$ & $+02: 04: 52.22$ & $2.1 \pm 0.1 \pm 0.1$ & $58.5 \pm 2.8 \pm 5.6$ & $57.6 \pm 4.9 \pm 12.4$ & $1.8 \pm 0.3 \pm 0.7$ \\
\hline M10 & 3.5 & $16: 57: 08.981$ & $-04: 06: 00.44$ & $1.0 \pm 0.1 \pm 0.1$ & $63.5 \pm 9.0 \pm 14.7$ & $111.1 \pm 48.9 \pm 38.7$ & $0.6 \pm 0.3 \pm 0.5$ \\
\hline M12 & 5.3 & $16: 47: 14.190$ & $-01: 56: 53.36$ & $0.5 \pm 0.1 \pm 0.1$ & $178.9 \pm 9.9 \pm 19.3$ & $115.8 \pm 48.8 \pm 83.8$ & $0.4 \pm 0.4 \pm 0.7$ \\
\hline M13 & 2.7 & $16: 41: 41.147$ & $+36: 27: 36.62$ & $1.7 \pm 0.1 \pm 0.1$ & $106.5 \pm 3.6 \pm 7.8$ & $115.8 \pm 3.8 \pm 16.8$ & $1.8 \pm 0.3 \pm 1.1$ \\
\hline M28 & 2.2 & $18: 24: 32.878$ & $-24: 52: 13.74$ & $2.1 \pm 0.2 \pm 0.2$ & $46.4 \pm 6.5 \pm 16.7$ & $57.5 \pm 10.3 \pm 34.9$ & $1.4 \pm 0.6 \pm 3.0$ \\
\hline M53 & 3.7 & $13: 12: 55.208$ & $+18: 10: 06.22$ & $1.0 \pm 0.2 \pm 0.1$ & $22.5 \pm 12.6 \pm 19.2$ & $54.4 \pm 4.2 \pm 10.4$ & $1.3 \pm 0.2 \pm 0.6$ \\
\hline M56 & 5.3 & $19: 16: 35.630$ & $+30: 11: 01.44$ & $0.8 \pm 0.1 \pm 0.1$ & $34.9 \pm 14.7 \pm 19.5$ & $69.5 \pm 7.5 \pm 11.6$ & $1.4 \pm 0.3 \pm 0.5$ \\
\hline M80 & 2.7 & $16: 17: 02.403$ & $-22: 58: 34.12$ & $2.3 \pm 0.1 \pm 0.1$ & $139.0 \pm 3.7 \pm 8.5$ & $155.4 \pm 17.5 \pm 20.7$ & $0.9 \pm 0.2 \pm 0.5$ \\
\hline M92 & 2.7 & $17: 17: 07.383$ & $+43: 08: 09.23$ & $1.6 \pm 0.2 \pm 0.1$ & $8.9 \pm 5.0 \pm 12.0$ & $55.3 \pm 4.7 \pm 4.4$ & $1.7 \pm 0.2 \pm 0.5$ \\
\hline NGC 6934 & 5.3 & $20: 34: 11.346$ & $+07: 24: 16.95$ & $1.8 \pm 0.1 \pm 0.1$ & $170.9 \pm 3.9 \pm 11.9$ & $144.9 \pm 66.2 \pm 84.7$ & $0.2 \pm 0.3 \pm 0.5$ \\
\hline
\end{tabular}

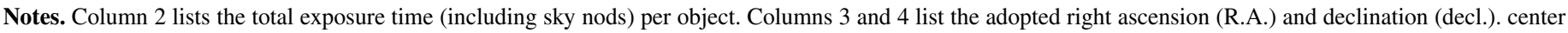

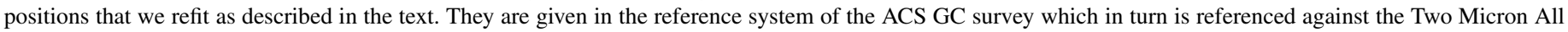

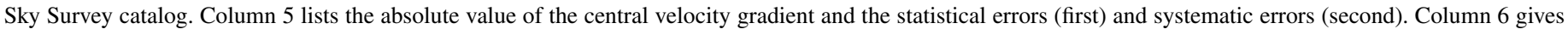

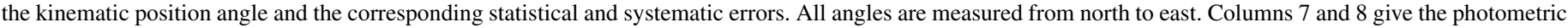

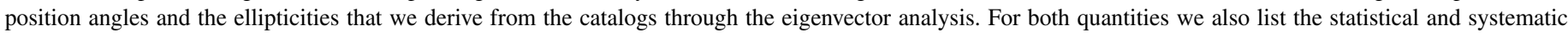

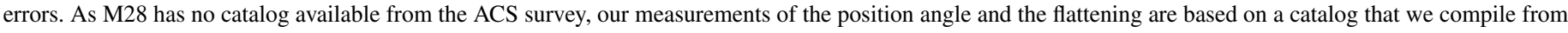
$H S T$ archival data as described in the text.

east-west axis. The fibers are 3 ". 2 in diameter on sky and are arranged in a dense-pack configuration with a fill-factor of $1 / 3$ such that three dithered observations fill in the gaps between fibers.

For each cluster we observed two offset but slightly overlapping pointings such that the combined field of view amounts to about $105^{\prime \prime} \times 105^{\prime \prime}$. Depending on surface brightness, we took 600-2400 s exposures in each dither position (see Table 1), split in half for cosmic ray rejection. We took $600 \mathrm{~s}$ empty sky exposures between each dither position and recorded bias frames and $\mathrm{Hg}$ and $\mathrm{Ne}$ arc lamp exposures for calibration every night.

\section{DATA REDUCTION AND KINEMATIC EXTRACTION}

Our data reduction uses the fitstools package by Gössl \& Riffeser (2002) and the Cure pipeline developed by our group for HETDEX (Hill et al. 2004). The procedure follows standard prescriptions for the generation of master biases, flats and arc lamp frames.

After the spectral extraction, we average the two cosmic ray split exposures and the bracketing sky exposures, rejecting spurious events. We scale the sky spectra by exposure time and subtract them from the science exposures. We combine the individual fiber spectra of the two dithered pointings into one datacube by imposing a pixel grid with an edge length of 1". 6 . A detailed description of the reduction is given in Fabricius et al. (2014).

Given the 3 ".2 fibers, we typically integrate over the light of several stars per fiber. A simple cross-correlation method may therefore yield uncertain velocities. We use a newly implemented version of the maximum penalized likelihood method by Gebhardt et al. (2000; see Fabricius et al. 2014) to first recover non-parametric line of sight velocity distributions (LOSVDs). We then fit Gaussian models to each LOSVD while only taking velocity channels that are separated by no more than $40 \mathrm{~km} \mathrm{~s}^{-1}$ from the systemic velocity into account. We extract the ten brightest spectra of each datacube to use as templates, since they are most likely dominated by the light of individual bright stars. We pick the spectrum that delivers the lowest rms of the residuals in the non-parametric fit as final template. We employ no spatial binning but reject spectra with a mean signal-to-noise below 5. Typical errors for the recovered mean velocities are $1.5 \mathrm{~km} \mathrm{~s}^{-1}$.

The derivation of velocities for each pixel produces a velocity field to which we fit a plane parameterized by

$$
v(x, y)=a x+b y+v_{\text {sys }},
$$

where the slopes $a$ and $b$ and the systemic velocity $v_{\text {sys }}$ are free parameters. The fit is carried out using a standard minimum least-squares fitting routine (MINPACK Imdif; Moré et al. 1980). From this, we obtain a kinematic position angle P.A.kin $=\arctan (b / a)$ and the absolute value of the central velocity gradient $\|\nabla v\|=\sqrt{a^{2}+b^{2}}$. During the fit, we reject outlier pixels (in velocity) through $\kappa-\sigma$ clipping with $\kappa=2.5$. The mean rms of the residuals of the plane fit is $3.3 \mathrm{~km} \mathrm{~s}^{-1}$ over all GCs. We estimate the statistical uncertainty through bootstrapping: we draw 100 subsamples with replacement and perform the plane fit for each of the subsamples. Individual pixels in the velocity fields are correlated over scales of up to 5 pixels because of fiber size and seeing. The bootstrapping method therefore does not reflect the systematic uncertainty caused by a few high-velocity stars. To assess this effect, we generate another 100 data sets where we mask a total of 11 randomly placed $7 \times 7$ pixel $^{2}$, subregions, covering about $10 \%$ of all pixels. We repeat the plane fit to all these 100 data sets and report the total spread of position angles and gradients as systematic uncertainty.

\section{PHOTOMETRIC POSITION ANGLES AND CENTRAL ELLIPTICITIES}

In order to test if the observed rotation is reflected in the morphology, we derive ellipticities and position angles using published catalogs. These are based on data from the Advanced Camera for Surveys on board the Hubble Space Telescope (HST) and collected as part of the ACS survey of GCs (Sarajedini et al. 2007). We obtain the ellipticities and position angles from an 

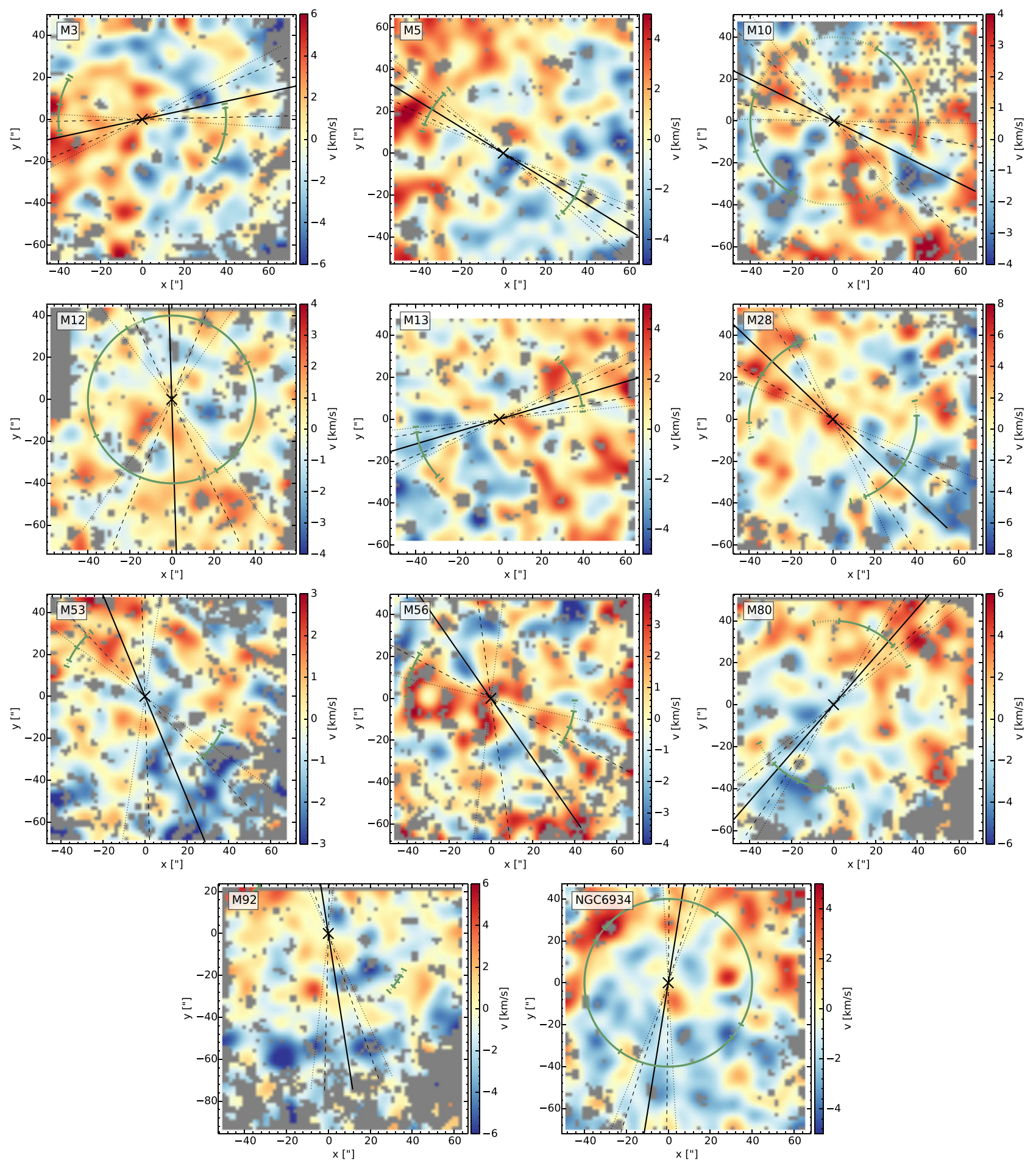

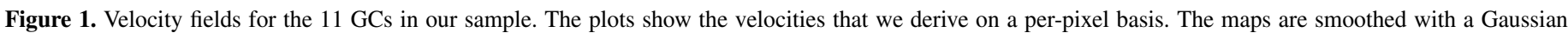

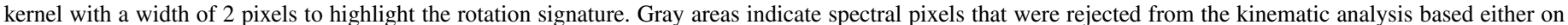

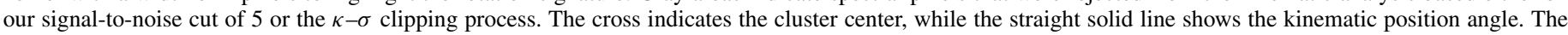

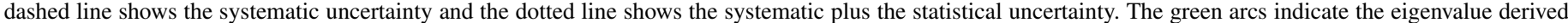

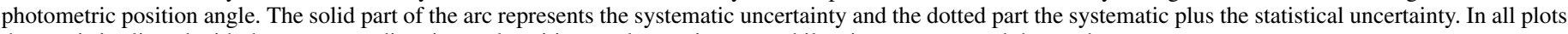
the $x$-axis is aligned with the east-west direction and positive $x$ values point west while $y$ increases toward the north.

eigenvector analysis of the spatial star distribution. For this we compute the $2 \times 2$ covariance matrix of the stellar positions as

$$
V_{x, y}=\operatorname{cov}(x, y)=\left\langle\left(x-\mu_{x}\right)\left(y-\mu_{y}\right)\right\rangle,
$$

where $x$ and $y$ are the catalog coordinates and $\mu_{x}$ and $\mu_{y}$ are the adopted center coordinates of the cluster. The eigenvectors $v_{1}, v_{2}$ and the eigenvalues $\lambda_{1}, \lambda_{2}$ then yield the ellipticity and the position angle through $\varepsilon=1-\sqrt{\lambda_{2} / \lambda_{1}}$ and P.A. $=$ $\arctan \left(v_{1, y} / v_{1, x}\right)$. We reject stars outside a radius of $100^{\prime \prime}$ which reaches the edge of the field of view of ACS-Wide Field Channel. The catalogs exclude non-cluster members.

We compute the center positions iteratively by shifting the $r=100^{\prime \prime}$ aperture until the first moments in both spatial directions become zero with respect to the new center position. 

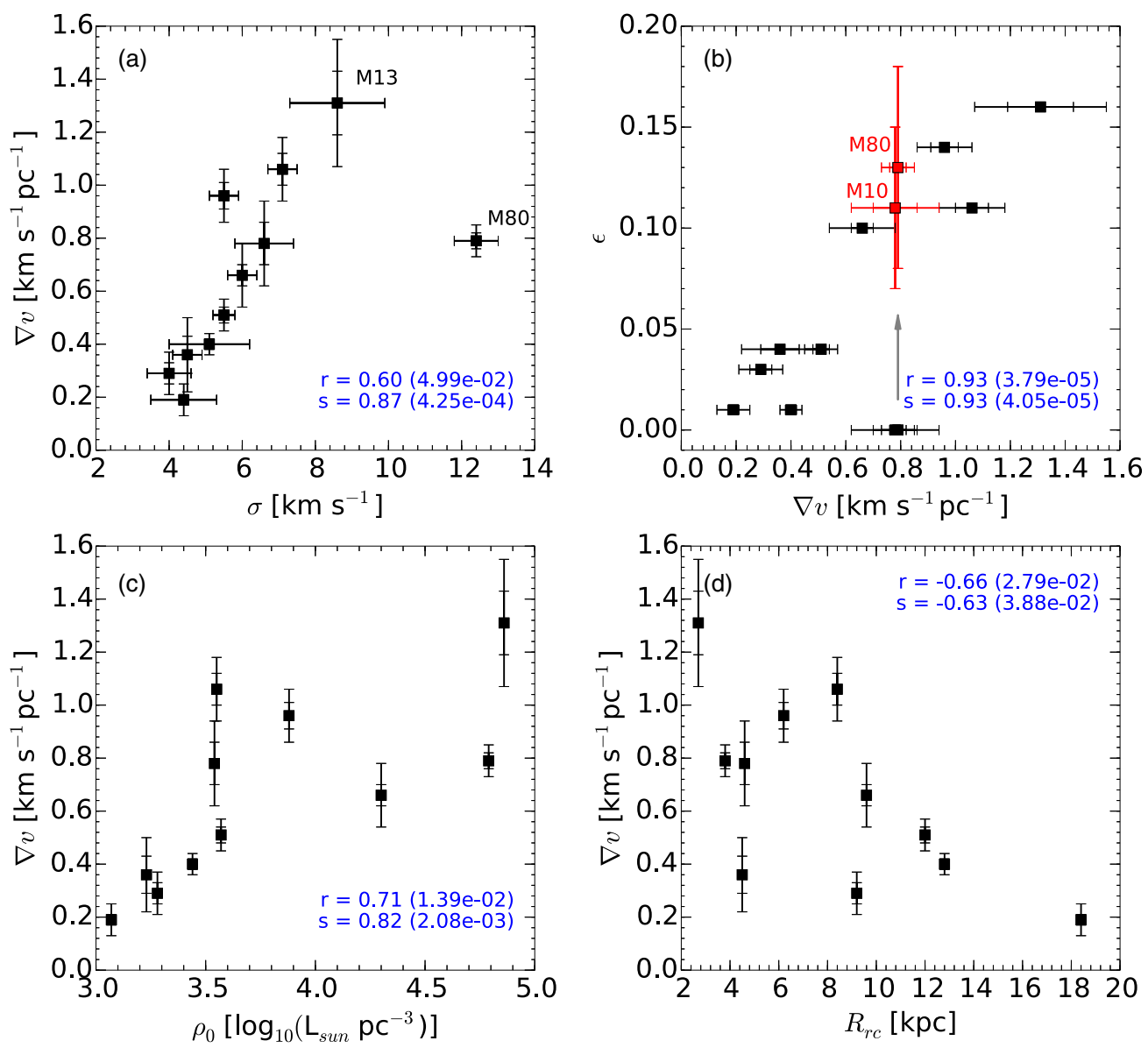

Figure 2. Correlations with parameters from the H96 catalog. (a) Central velocity gradient $\nabla v$ as function of the central velocity dispersion from H96. (b) Ellipticity as a function of $\nabla v$. For the two clusters M10 and M80 we plot the ellipticities from Chen \& Chen (2010) with red symbols. (c) $\nabla v$ plotted as a function of the central luminosity density. (d) $\nabla v$ plotted as function of the distance from the Galactic center. The Pearson correlation coefficient $r$ and the Spearman's rank correlation $s$ are shown in all panels. The corresponding two-tailed $p$-values are given in the parenthesis. For the ellipticity correlation $r$ and $s$ are computed with the updated values for M10 and M80 from Chen \& Chen (2010).

We compare these centers with the ones derived by Noyola \& Gebhardt (2006) for the common objects. The differences are under $2^{\prime \prime}$ on average. We tested that the aperture truncation does not affect our reported ellipticities. Using radial bin counts, we have further tested that the derived ellipticities and position angles are not caused by artifacts in the catalogs such as lower star counts around bright stars.

In general, our central ellipticities are small compared to published values at larger radii by up to a factor of 10 (compared, for instance, with Geyer et al. 1983; White \& Shawl 1987; Chen $\&$ Chen 2010). Also, we find no obvious correlation between our results and published ellipticities. This however is easily explained by the fact that our data probe vastly different radial scales.

As further verification of our derived position angles, we generate synthetic images from the catalogs by assigning the same magnitude to the stars in the catalog and adding them to an empty image with a point-spread function obtained from the ACS image itself. We then apply a Gaussian smoothing of $20^{\prime \prime}$. We overplot contours and verify visually that the eigenvector based position angles are not affected by artifacts in the catalogs.

We estimate the statistical uncertainty using the bootstrapping method in the same manner as we do for the kinematic data sets. The bin maps show that artifacts in the catalogs can affect our measurements of the ellipticity. We therefore run our analysis on hemispheres by mirroring all points of one hemisphere about the center onto the other side. We repeat this on 36 different hemispheres, separated by $10^{\circ}$. We report the total spread of values for the position angle and the ellipticity as systematic error.

The eigenvalue derived ellipticities show no significant values different from zero inside the VIRUS-W field of view, and consequently, the derived position angles are poorly constrained. The strongest signal is typically found in the $75^{\prime \prime}-100^{\prime \prime}$ radial range.

M28 is not part of the HST ACS survey; we therefore construct a catalog using DAOPHOT (Stetson 1987) on an archival HST ACS F625W image (PI: Jonathan Grindlay; see Noyola \& Gebhardt 2006 for a description of the process). We fill the gaps between the ACS chips and extend the coverage of the catalog in radial range using WFC3/UVIS F390W (PI: Francesco Ferraro) and WFPC2 F555W (PI: Roberto Buonanno) images. The final catalog extends to a radius of $85^{\prime \prime}$ from the cluster center.

\section{RESULTS}

Figure 1 shows maps of the line-of-sight velocity for the 11 clusters in our sample. The rotation is clearly seen as a velocity gradient across the field. We detect statistically significant central rotation in all clusters.

We translate the projected absolute values of the velocity gradients to physical units using distance estimates, core radii, 


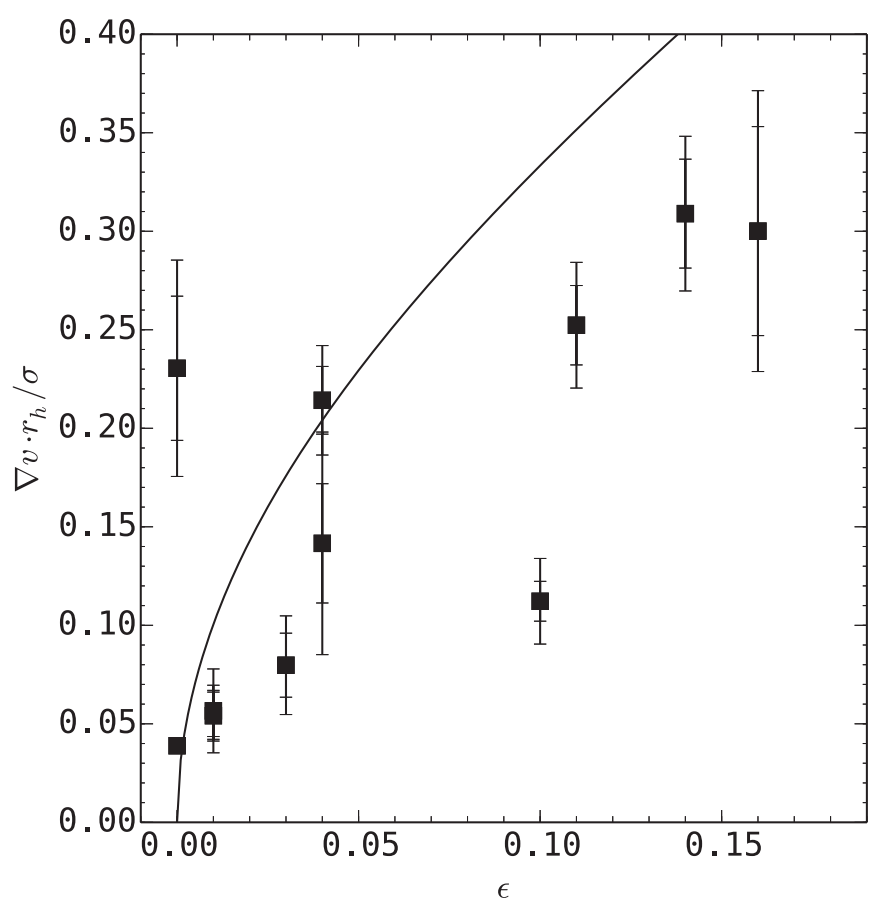

Figure 3. $(V / \sigma, \epsilon)$ diagram for the GCs in our sample. As proxy for the maximum rotational velocity we multiply our derived velocity gradients by the half-light radii from $\mathrm{H} 96$. The ellipticities are also taken from the same catalog. The solid line shows the location for isotropic rotators (Binney \& Tremaine 1987). Shorter error bars indicate the systematic errors, longer error bars the systematic plus the random errors.

and half-light radii from $\mathrm{H} 96$. We find that the velocity gradients have absolute values in the range of $0.2-1.3 \mathrm{~km} \mathrm{~s}^{-1} \mathrm{pc}^{-1}$ with average values of $0.7 \mathrm{~km} \mathrm{~s}^{-1} \mathrm{pc}^{-1}, 0.6 \mathrm{~km} \mathrm{~s}^{-1} r_{c}{ }^{-1}$, and $2.2 \mathrm{~km} \mathrm{~s}^{-1} r_{h}{ }^{-1}$.

In Figure 1 we indicate the derived kinematic position angles and also overplot the eigenvector derived position angles. The low ellipticities of M10, M12, and NGC 6934 leave the values from our eigenvalue analysis poorly constrained. In the other cases, both position angle measurements agree well within the errors with one notable exception: for M92 the kinematic position angle deviates from the catalog derived value by about $45^{\circ}$. Unfortunately, in the case of M92 the southern field covered a relatively sparse region of the cluster and thus, it poorly constrains the direction of the velocity gradient. We give the absolute values of the central velocity gradients, the kinematic and the eigenvector based position angles, adopted cluster centers, and the total exposure times in Table 1.

In Figure 2 we compare the absolute values of the central velocity gradients with other parameters from the $\mathrm{H} 96$ catalog. We find a very tight correlation with the central velocity dispersion, with the exception of M80. This correlation is possibly the result of dispersion measurements that include (and do not correct for) rotation. For example, M13 has the second largest published value of the central velocity dispersion in our sample (Cohen \& Meléndez 2005). By extrapolating the central rotation gradient to the actual positions of the stars we can derive a velocity dispersion of $9.2 \mathrm{~km} \mathrm{~s}^{-1}$. This extrapolation does not take the flattening of the rotation curve into account and therefore it is not surprising that this value is actually larger than the Cohen \& Meléndez (2005) value of $7.1 \mathrm{~km} \mathrm{~s}^{-1}$. It does show, however, that rotation can significantly impact the dispersion measurements. For M80, the rotation can probably not explain the large value of the central dispersion of $12.4 \mathrm{~km} \mathrm{~s}^{-1}$ (Dubath et al. 1997).

The observed correlation between the outer ellipticity and the central rotation however is most likely physical. The only outliers (M10 and M80) fall nicely on the correlation when the ellipticity values from H96 are replaced by those of Chen \& Chen (2010).

We also find a correlation between central luminosity density and the value of the velocity gradient. More speculative is a possible correlation of the amount of central rotation with the distance from the galactic center as shown in the lower right panel. If the flattening does increase toward the galactic center as suggested by White \& Shawl (1987) and Chen \& Chen (2010) and the flattening is as closely related to rotation as we find, then this may be expected.

With estimates for ellipticities, rotation, and velocity dispersion, we plot our objects in the $(V / \sigma, \epsilon)$ diagram (Figure 3$)$. The values for the rotation are obtained by multiplying the value of the central velocity gradient by the half-light radius $r_{h}$ from $\mathrm{H} 96$. The solid line indicates the locus of edge-on isotropic oblate rotators (Binney \& Tremaine 1987). All but two of our objects fall below this line. However, as discussed before, the estimates for the central dispersion are likely affected by rotation and also the extrapolation of the rotational velocity to $r_{h}$ may yield incorrect values for the actual maximum rotation. On the other hand our analysis does not take projection effects into account which may move objects even further away from the line. While we have no reason to expect that GCs behave like isotropic oblate rotators, the clusters in our sample follow a general trend of larger $(V / \sigma)$ for increased ellipticity which again indicates that rotation is the driving factor for the flattening.

\section{CONCLUSIONS}

We present central velocity fields for 11 Milky Way GCs derived from data collected in an ongoing survey using the fiber based, optical IFU instrument VIRUS-W. We find that all clusters presented here show significant gradients in the observed velocity fields that are indicative of rotation.

This is surprising and clearly shows a need for theoretical models to produce and to sustain rotation on scales comparable to the core radius. We also find that the orientation of the central flattening in stellar density (within an 100" aperture) is generally in good agreement with the kinematic position angle that we derive from the velocity fields.

We show that the central rotation correlates very well with published values for the central velocity dispersion, thus pointing to a possible impact from the rotation. A detailed analysis of this effect and a comparison with dispersion estimates from our data will be included in a subsequent publication. We also find a strong correlation with outer ellipticity indicating that, at least for the objects in our sample, the flattening is primarily due to rotation rather than the Milky Way tidal field.

There is an indication of an increase of rotation toward the galactic center. If true, this might have a strong impact on formation theories and clearly shows the need for a larger sample.

One caveat of our study so far is that our sample so far lacks any core-collapsed clusters. It is conceivable that this process will eradicate rotation through the transport of angular momentum to the outskirts of the cluster. Future observations will specifically target such systems. Our survey of 27 clusters will allow us to probe in detail how rotations correlate with properties such as central velocity dispersions, ellipticity, ages, spatial 
distribution, and total luminosity. This will complement both upcoming higher spatial resolution studies with instruments such as PMAS at the Calar Alto $3.5 \mathrm{~m}$ (Kamann et al. 2014) and MUSE at the Very Large Telescope, and multi-object spectroscopic studies that are limited to much larger radii. Our derived position angles will allow the multi-object studies to specifically target angular ranges of maximum rotation.

We thank the anonymous referee for the thoughtful comments. This research has made use of the NASA/IPAC Extragalactic Database (NED) which is operated by the Jet Propulsion Laboratory, California Institute of Technology, under contract with the National Aeronautics and Space Administration. Some of the data presented in this Letter were obtained from the Mikulski Archive for Space Telescopes (MAST). STScI is operated by the Association of Universities for Research in Astronomy, Inc., under NASA contract NAS5-26555. Support for MAST for non-HST data is provided by the NASA Office of Space Science via grant NNX13AC07G and by other grants.

\section{REFERENCES}

Anderson, J., \& King, I. R. 2003, AJ, 126, 772

Bekki, K. 2010, ApJL, 724, L99

Bellazzini, M., Bragaglia, A., Carretta, E., et al. 2012, A\&A, 538, A18

Bianchini, P., Varri, A. L., Bertin, G., \& Zocchi, A. 2013, ApJ, 772, 67

Binney, J., \& Tremaine, S. 1987, Galactic Dynamics (Princeton, NJ: Princeton Univ. Press)
Chen, C. W., \& Chen, W. P. 2010, ApJ, 721, 1790

Cohen, J. G., \& Meléndez, J. 2005, AJ, 129, 303

Dubath, P., Meylan, G., \& Mayor, M. 1997, A\&A, 324, 505

Einsel, C., \& Spurzem, R. 1999, MNRAS, 302, 81

Ernst, A., Glaschke, P., Fiestas, J., Just, A., \& Spurzem, R. 2007, MNRAS, 377,465

Fabricius, M. H., Coccato, L., Bender, R., et al. 2014, arXiv:1404.2272

Fabricius, M. H., Grupp, F., Bender, R., et al. 2012, Proc. SPIE, 8446, 84465K

Gebhardt, K., Richstone, D., Kormendy, J., et al. 2000, AJ, 119, 1157

Geyer, E. H., Nelles, B., \& Hopp, U. 1983, A\&A, 125, 359

Gössl, C. A., \& Riffeser, A. 2002, A\&A, 381, 1095

Harris, W. E. 1996, AJ, 112, 1487

Hill, G. J., Gebhardt, K., Komatsu, E., \& MacQueen, P. J. 2004, in AIP Conf. Proc. 743, The New Cosmology: Conference on Strings and Cosmology, ed. R. E. Allen, D. V. Nanopoulos, \& C. N. Pope (Melville, NY: AIP), 224

Kamann, S., Wisotzki, L., Roth, M. M., et al. 2014, arXiv:1404.2781

Lane, R. R., Kiss, L. L., Lewis, G. F., et al. 2011, A\&A, 530, A31

Longaretti, P.-Y., \& Lagoute, C. 1997, A\&A, 319, 839

Mastrobuono-Battisti, A., \& Perets, H. B. 2013, ApJ, 779, 85

Meylan, G., \& Heggie, D. C. 1997, A\&ARv, 8, 1

Moré, J. J., Garbow, B. S., \& Hillstrom, K. E. 1980, User Guide for MINPACK-1 (Report ANL-80-74; Argonne, IL: Argonne National Laboratory)

Noyola, E., \& Gebhardt, K. 2006, AJ, 132, 447

Sarajedini, A., Bedin, L. R., Chaboyer, B., et al. 2007, AJ, 133, 1658

Stetson, P. B. 1987, PASP, 99, 191

Trager, S. C., King, I. R., \& Djorgovski, S. 1995, AJ, 109, 218

van den Bosch, R., de Zeeuw, T., Gebhardt, K., Noyola, E., \& van de Ven, G. 2006, ApJ, 641, 852

van Leeuwen, F., \& Le Poole, R. S. 2002, in ASP Conf. Ser. 265, Omega Centauri, A Unique Window into Astrophysics, ed. F. van Leeuwen, J. D. Hughes, \& G. Piotto (San Francisco, CA: ASP), 41

White, R. E., \& Shawl, S. J. 1987, ApJ, 317, 246

Zocchi, A., Bertin, G., \& Varri, A. L. 2012, A\&A, 539, A65 